\title{
Searches for additional Higgs bosons decaying to tau leptons at the LHC
}

C. Caputo ${ }^{1}$, on behalf of the $A T L A S$ an $C M S$ collaborations.

1 Centre for Cosmology, Particle Physics and Phenomenology, Université catholique de Louvain, Louvain-la-Neuve B-1348, Belgium

* claudio.caputo@cern.ch

December 21, 2018

Proceedings for the 15th International Workshop on Tau Lepton Physics, Amsterdam, The Netherlands, 24-28 September 2018 scipost.org/SciPostPhysProc. Tau2018

\section{Abstract}

The searches for additional Higgs bosons decaying to tau leptons in scenarios beyond the standard model will be summarised, from the pp collision data collected by the ATLAS and CMS experiments at LHC Run-2.

\section{Contents}

$\begin{array}{lll}1 & \text { Introduction } & 1\end{array}$

\begin{tabular}{|lll|}
\hline 2 & Search for a neutral MSSM Higgs boson decaying into $\tau \tau$ & 2
\end{tabular}

\begin{tabular}{|lll}
3 & Search for charged Higgs bosons with the $H^{ \pm} \rightarrow \tau^{ \pm} \nu$ & 6
\end{tabular}

$4 \quad$ Search for new light bosons in decays of the $h(125) \quad 9$

$4.1 \quad h \rightarrow a a \rightarrow b \bar{b} \tau \tau) \quad 9$

$\begin{array}{llr}4.2 \quad h \rightarrow a a \rightarrow \mu \mu \tau \tau & 9\end{array}$

\begin{tabular}{llr}
\hline \hline 4.3 & Results & 10
\end{tabular}

$\begin{array}{lll}5 \text { Conclusion } & 10\end{array}$

\begin{tabular}{lr}
\hline References & 12
\end{tabular}

\section{Introduction}

The discovery of a new particle in July 2012 by the ATLAS [1] and CMS [2] collaborations at the Large Hadron Collider (LHC) [3] [4, 5], compatible with the standard model (SM) Higgs boson, is a fundamental step forward in our understanding of the electroweak spontaneous symmetry breaking. However, many open questions, including the problem 
of the large hierarchy between the Plack and electroweak scale, still need to be addressed. In order to cope with this, many different extension of the SM have been proposed, like supersymmetry (SUSY) 6, 7].

Extending the SM entails, in most of the cases, the extension of the Higgs sector. One of the simple extensions is described by the 2 Higgs Doublet Model (2HDM), where two scalar Higgs doublets are introduced. The spontaneous symmetry breaking give rise to five scalar bosons: a neutral CP-odd $A$, two neutral CP-even $h$ and $H$, two charged bosons $H^{ \pm}$. In the decoupling limit, the lightest scalar of $2 \mathrm{HDM}$ can have properties compatible with discovered Higgs boson; in this scenario all other scalars have larger masses.

Considering how the two doublets can interact with other particles of the SM, different phenomenology scenarios can appear. One of this scenarios is the Type-II 2HDM, which supposes that the first doublet couples only with up-quarks, while the second doublet only with down-quarks and charged fermions.

The Minimal Supersymmetric Standard Model (MSSM) [8, 9], which incorporate the supersymmetry, is a Type-II 2HDM. At tree level, all the phenomenology can be described by two parameters, conventionally chosen to be the mass of the pseudoscalar Higgs $m_{A}$ and the ratio between the two vacuum expectation values (VEVs) $\tan \beta=\nu_{1} / \nu_{2}$.

For $A$ and $H$ the dominant production process is still the gluon fusion, for small and medium values of $\tan \beta$, followed by the $b \bar{b}$-associated production, that increase at high $\tan \beta$ due to the second doublet couplings to down-type fermions. The $H^{ \pm}$production mechanism is strictly connected to the mass of the charged boson. For mass below the top-quark mass $\left(m_{H^{+}}<m_{t}\right)$ the decay mode in a $\tau$ lepton plus is neutrino dominate in a Type-II $2 \mathrm{HDM}$ scenario; for mass above the top-quark mass $\left(m_{H^{+}}>m_{t}\right)$, decay mode $\tau \nu$ increase with $\tan \beta$. In this report, results of direct searches of MSSM Higgs bosons with tau leptons in the final state, from the ATLAS and CMS collaborations using the 2016 dataset, are presented.

A complex $S U(2)_{L}$ singlet field $S$ can be added to $2 \mathrm{HDM}$, with a small mixing with the doublets; such a model is called $2 \mathrm{HDM}+\mathrm{S}$. This leads to two additional singlet states, a CP-odd scalar $a$ and a CP-even $s$, which inherit a mixture of the Higgs doublets fermion interactions. In such a model, also known as NMSSM, the branching fraction of the Higgs boson to a pair of $a$ or $s$ bosons can be sizeable, and a wide variety of exotic Higgs decays are allowed [10], especially $h \rightarrow a a$. In this report, results of direct searches of $h \rightarrow a a$ with tau leptons in the final state, from the CMS collaborations using the 2016 dataset, are presented.

\section{Search for a neutral MSSM Higgs boson decaying into $\tau \tau$}

The coupling of the $\mathrm{H}$ and the $\mathrm{A}$ to down-type fermions, at leading-order ( $\mathrm{LO}$ ), is enhanced by $\tan \beta$ with respect to the expectation for an SM Higgs boson of the same mass, while the coupling to vector bosons and up-type fermions is suppressed. The enhanced coupling to down-type fermions makes searches for additional heavy neutral Higgs bosons that exploit final states containing $\tau \tau$ particularly interesting. It also has consequences for the production: firstly, the production in association with b quarks dominates over the production via gluon fusion for large values of $\tan \beta$. Secondly, in gluon fusion production the kinematic properties of the Higgs boson change as a function of tan $\mathrm{b}$ due to the increasing contribution of b quarks in the fermion loop. Diagrams for $h, H$, and $A$ production at LO are shown in Figure 1 .

The ATLAS and CMS collaborations performed the direct search in the most sensitive final states of the taus [11] [12]. Both focus their attention on $e \tau_{h}, \mu \tau_{h}$ and $\tau_{h} \tau_{h}$, where $\tau_{h}$ 

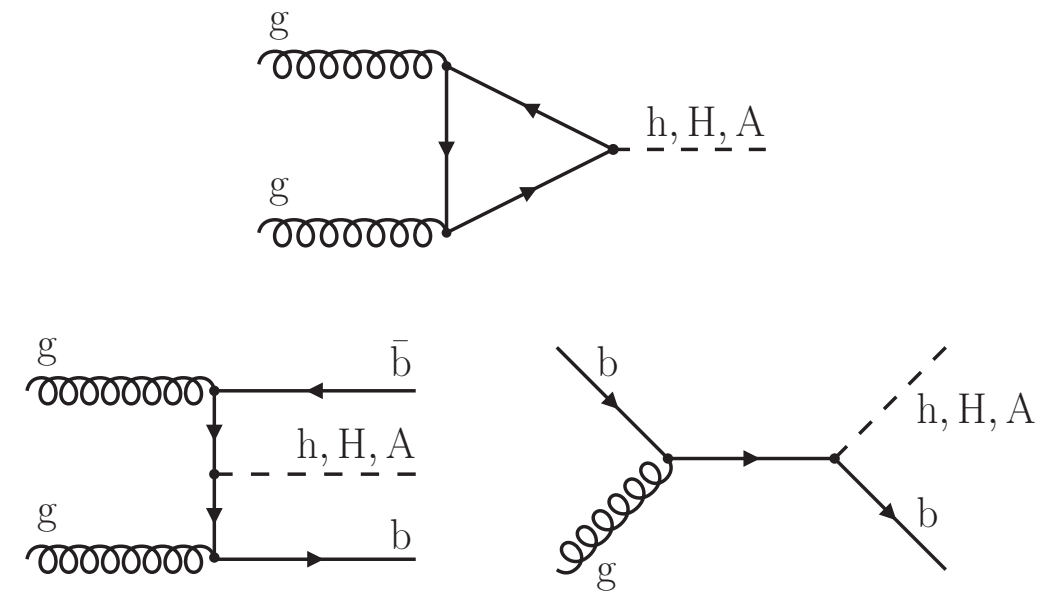

Figure 1: Feynman diagrams of the production modes of an neutral MSSM Higgs boson. (top) Gluon gluon fusion (bottom-left) $b \bar{b}$-associated production four-flavour scheme (bottom-right) $b \bar{b}$-associated production five-flavour scheme

indicates a tau decaying hadronically; CMS consider also the $e \mu$ final state. The dataset analyzed corresponds to the full statistics available from 2016 collisions, $\sim 36 \mathrm{fb}^{-1}$, at center-of-mass energy of $13 \mathrm{TeV}$. Events are categorized in order to exploit the topological kinematic peculiarities of MSSM production mechanisms. The categories depend whether a b-jet is found in the event, in order to select the $b \bar{b}$-associated production if a b-jet is present (b-tag), or select the gluon fusion production if no b-jet is found (b-veto). Further sub-categorization are performed to add more control regions used for constraining particular backgrounds.

The dominant background contribution comes from misidentification of jets as $\tau_{h}$, which is estimated using a data-driven technique called Fake-Factor Method. This method is extensively explained in [11,12]. Other important background contributions come from $Z / \gamma^{*} \rightarrow \tau \tau$ production in the b-veto category, $t \bar{t}$ production in the b-tag category, and to a lesser extent $W(\rightarrow l \nu)+$ jets, single top-quark, diboson and $Z(\rightarrow l l)+$ jets production. These contributions are estimated using simulation, in some cases re-normalized using control regions in data. Corrections are applied to the simulation to account for mismodelling of the trigger, reconstruction, identification and isolation efficiency, the electron to $\tau_{h}^{\text {vis }}$ misidentification rate and the momentum scales and resolutions.

The total transverse mass of the system is used as final discriminant to search for an excess due to signal,

$$
m_{T}^{\text {tot }}=\sqrt{m_{T}^{2}\left(\tau_{1}, \tau_{2}\right)+m_{T}^{2}\left(\tau_{1}, E_{T}^{\text {miss }}\right)+m_{T}^{2}\left(\tau_{2}, E_{T}^{\text {miss }}\right)},
$$

where $\tau_{1}$ and $\tau_{2}$ respectively refer to the $p_{T}$ leading and sub-leading taus, while $E_{T}^{\text {miss }}$ is the missing energy measured in the event considered. The $m_{T}^{\text {tot }}$ binned distribution is fitted simultaneously in all the categories used in the analysis. No evidence for a signal is found. Both collaborations set upper limits at 95\% confidence level (CL) on the crosssection times branching fraction for to two dominant production modes, gluon fusion and $b \bar{b}$-associated production. The limits are computed in the narrow width approximation. Figure 2 shows the upper limits obtained by the ATLAS and CMS collaborations as a function of Higgs boson mass.

Results are re-interpreted in two different benchmark scenario models; the $m_{h}^{\text {mod+ }}$ and the hMSSM scenarios 13, 14. Figure 3 shows limits set on $m_{A}-\tan \beta$ plane. 

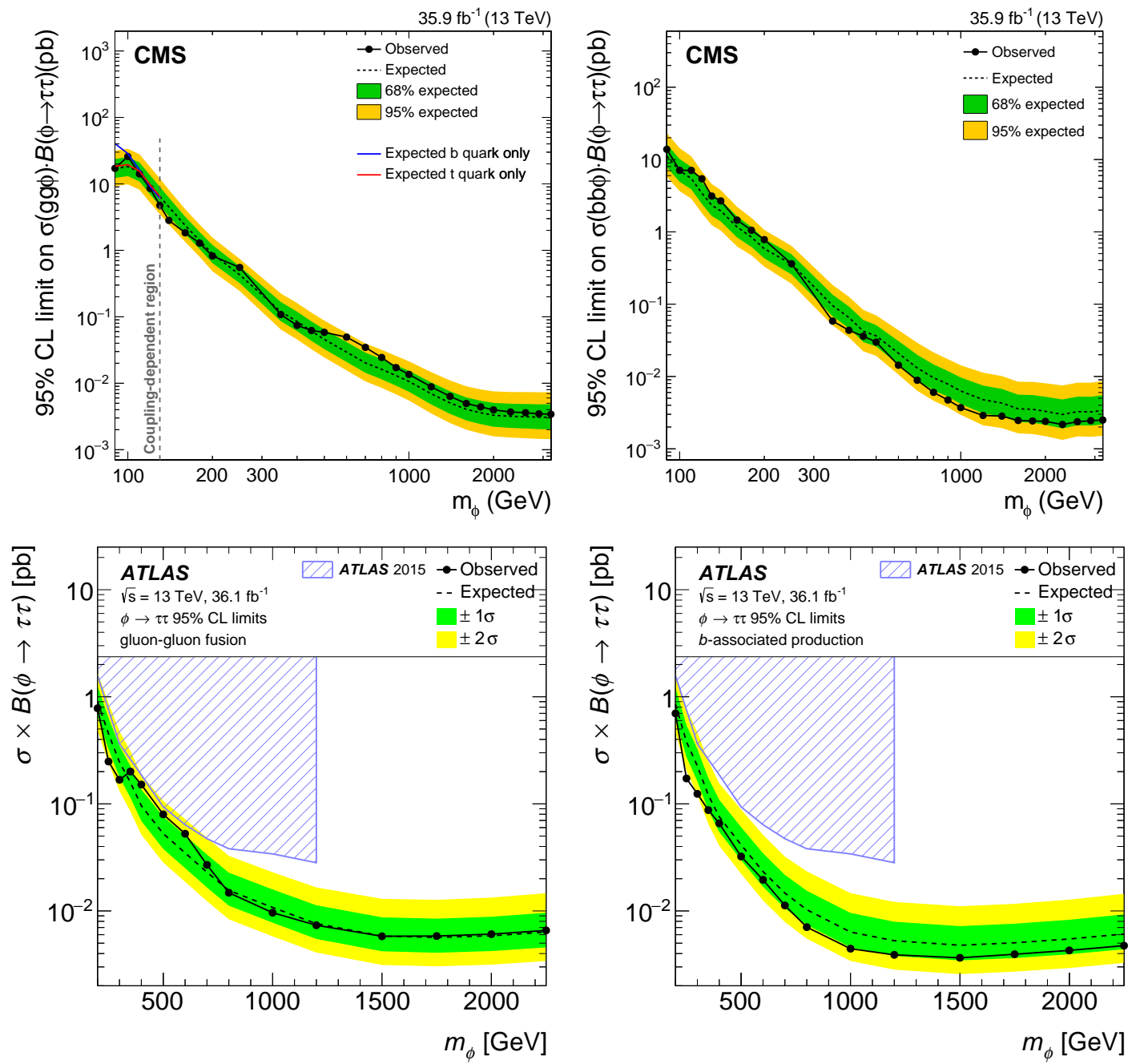

Figure 2: (top) ATLAS expected and observed limits on $\sigma(\phi) \times B R(\phi \rightarrow \tau \tau)$ for (left) the gluon fusion and (right) the $b \bar{b}$-associated production, resulting from the combination of all the three channels considered. (bottom) CMS expected and observed limits on $\sigma(\phi) \times B R(\phi \rightarrow \tau \tau)$ for (left) the gluon fusion and (right) the $b \bar{b}$-associated production, resulting from the combination of all the four channels considered. [11] 12] 

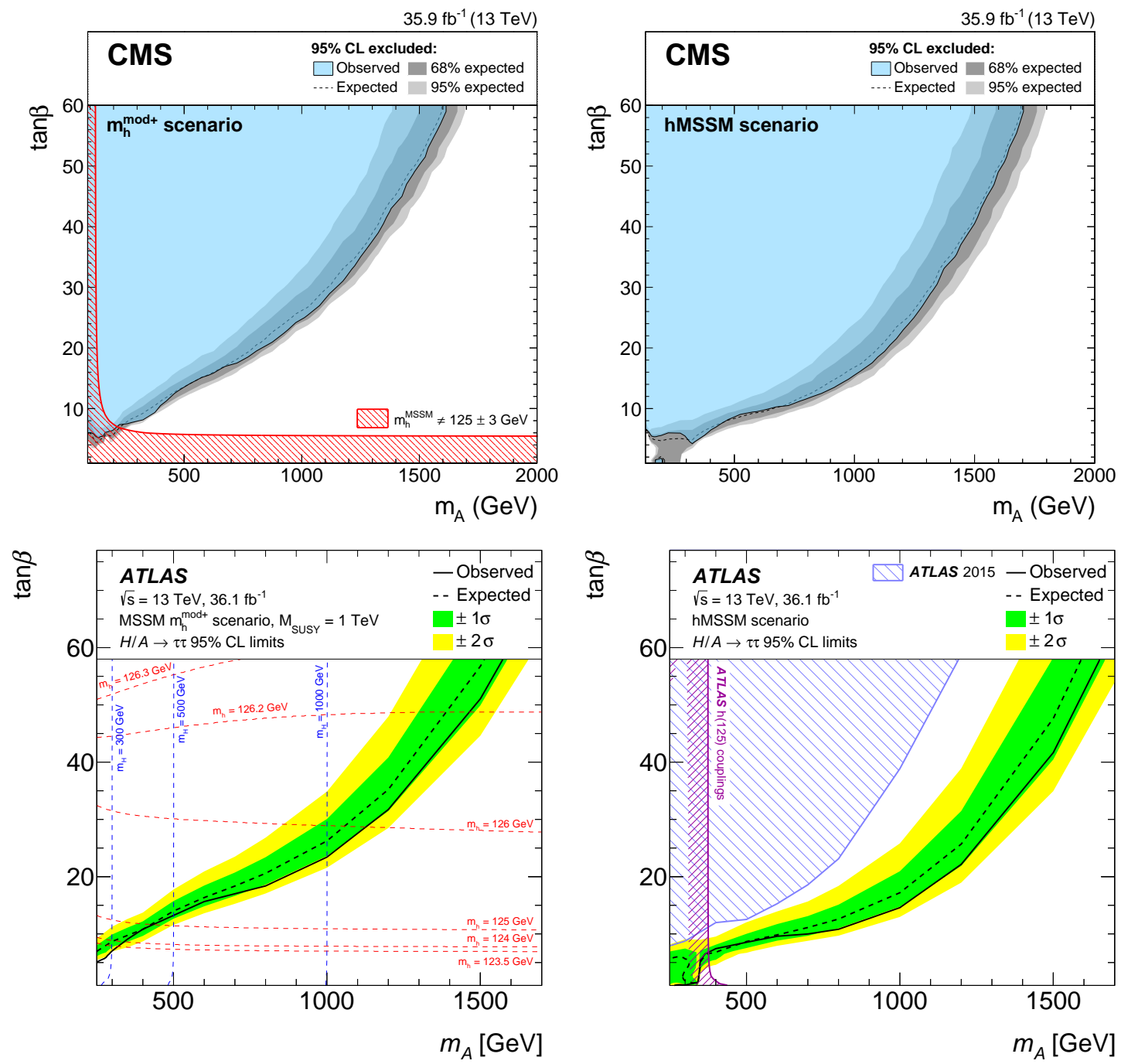

Figure 3: Model dependent exclusion limits in the $m_{A}-\tan \beta$ plane for the (left) $m_{h}^{\bmod +}$ and the (right) hMSSM scenarios. In the top left plot, the red shade area indicates the region that does not give a light $h$ higgs boson consistent with a mass of $125 \mathrm{GeV}$ within the theoretical uncertainties $\pm 3 \mathrm{GeV}$. In the bottom left plot, the red dashed lines represent the different parameters value that give a particular $m_{h}$ value. In the bottom right plot, the purple area indicates the region already excluded by constrains on $h(125)$ couplings. [11] 12] 


\section{Search for charged Higgs bosons with the $H^{ \pm} \rightarrow \tau^{ \pm} \nu$}

The $H^{ \pm}$production mechanism is strictly connected to the mass of the charged boson. If $H^{ \pm}$mass is below the top-quark mass $\left(m_{H^{+}}<m_{t}\right)$, the production mode goes through the decay of a top-quark, $t \rightarrow b H^{+}$, in a $t \bar{t}$ production. In this mass range, the decay mode in a $\tau$ lepton plus is neutrino dominate in a Type-II $2 \mathrm{HDM}$ scenario. If $H^{ \pm}$mass above the top-quark mass $\left(m_{H^{+}}>m_{t}\right)$, the dominant production mode is $g g \rightarrow t b H^{+}$. In this mass range, the dominant decay is $H^{+} \rightarrow t b$, considering the alignment limit $(\cos \beta-\alpha \simeq 0)[15] ;$ however the branching fraction for $H^{+} \rightarrow \tau \nu$ can reach up to $10 \div 15 \%$ at high $\tan \beta$. The mass region where the $H^{ \pm}$and the top-quark masses are similar $\left(m_{H^{+}} \simeq m_{t}\right)$ involves interference effects among the $t \bar{t}$ and $H^{ \pm}$non-resonant top-quark productions. Recently theoretical prediction become available for this region [16], which now allows to compare directly the $H^{ \pm}$model with data in proximity of the top-quark mass. In Figure 4 the different production modes Feynman diagrams are depicted.
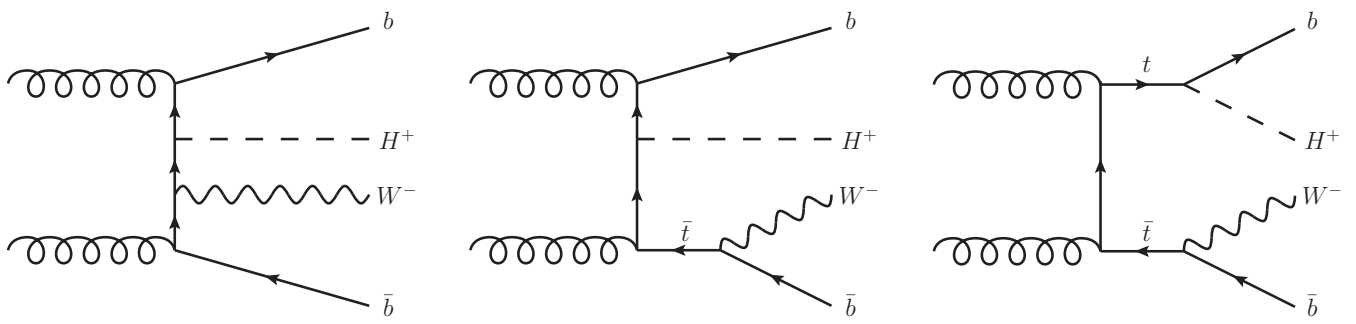

Figure 4: Examples of leading-order Feynman diagrams contributing to the production of charged Higgs bosons in pp collisions: (left) non-resonant top-quark production, (center) single-resonant top-quark production that dominates at large $H^{+}$masses, (right) double-resonant top-quark production that dominates at low $\mathrm{H}^{+}$masses. The interference between these three main diagrams becomes most relevant in the intermediate-mass region.

The ATLAS and CMS collaboration searched for a charged Higgs boson in pp collision using the full 2016 dataset, $\sim 36 \mathrm{fb}^{-1}$, at a center-of-mass energy of $13 \mathrm{TeV}$ 17] [18. The results presented will refer to the ATLAS search, the only one with the full 2016 dataset public at the time of the conference.

Two different channels are considered: $\tau_{h}+$ jets and $\tau_{h}+$ lepton, where both aim to different decays of the top-quark produced with the $H^{ \pm}$. Furthermore, a multivariate discriminant is used to increase the search sensitivity, exploiting the kinematic variables that differentiate between signal and backgrounds. The output score of a Boosted Decision Trees (BDTs) is used as final discriminant. In order to take advantage of the different $H^{ \pm}$decay products' kinematic regime, simulated signal sample are divide in five $H^{ \pm}$mass bins: $90-120 \mathrm{GeV}, 130-160 \mathrm{GeV}, 160-180 \mathrm{GeV}, 200-400 \mathrm{GeV}$ and $500-2000 \mathrm{GeV}$. The BDTs are trained using a set of variables related to the particular final state.

Backgrounds classification and estimation depends on the type of object that gives rise to the identified $\tau_{h}$. If $\tau_{h}$ arise from a true hadronically decaying tau or electron/muon misidentification, simulation is used to estimate such backgrounds like $Z+$ jets, $W+$ jets or dibosons; however, in the case of $t \bar{t}$ events, the normalization is obtained from a fit to the data. If $\tau_{h}$ arise from a misidentified gluon-jet or quark-jet, the Fake Factor Method is used to estimate such background [17]. Figure 5 shows the BDTs output for the $\tau_{h}+$ jets final state after estimating the different background contributions.

BDTs binned distribution are fitted simultaneously in all the three signal regions. The 
data are found to be consistent with the background-only hypothesis. Exclusion limits are set at $95 \% \mathrm{CL}$ on $\sigma\left(p p \rightarrow t b H^{+}\right) \times B\left(H^{+} \rightarrow \tau \nu\right)$ for the full mass range, as well as on $B\left(t \rightarrow b H^{+}\right) \times B\left(H^{+} \rightarrow \tau \nu\right)$ for low mass range. Figure 6 shows the expected and observed exclusion limits as a function of the $H^{ \pm}$mass hypothesis. Figure 7 shows $95 \%$ CL exclusion limits on $\tan \beta$ as a function of the charged Higgs boson mass in the context of the hMSSM scenario.
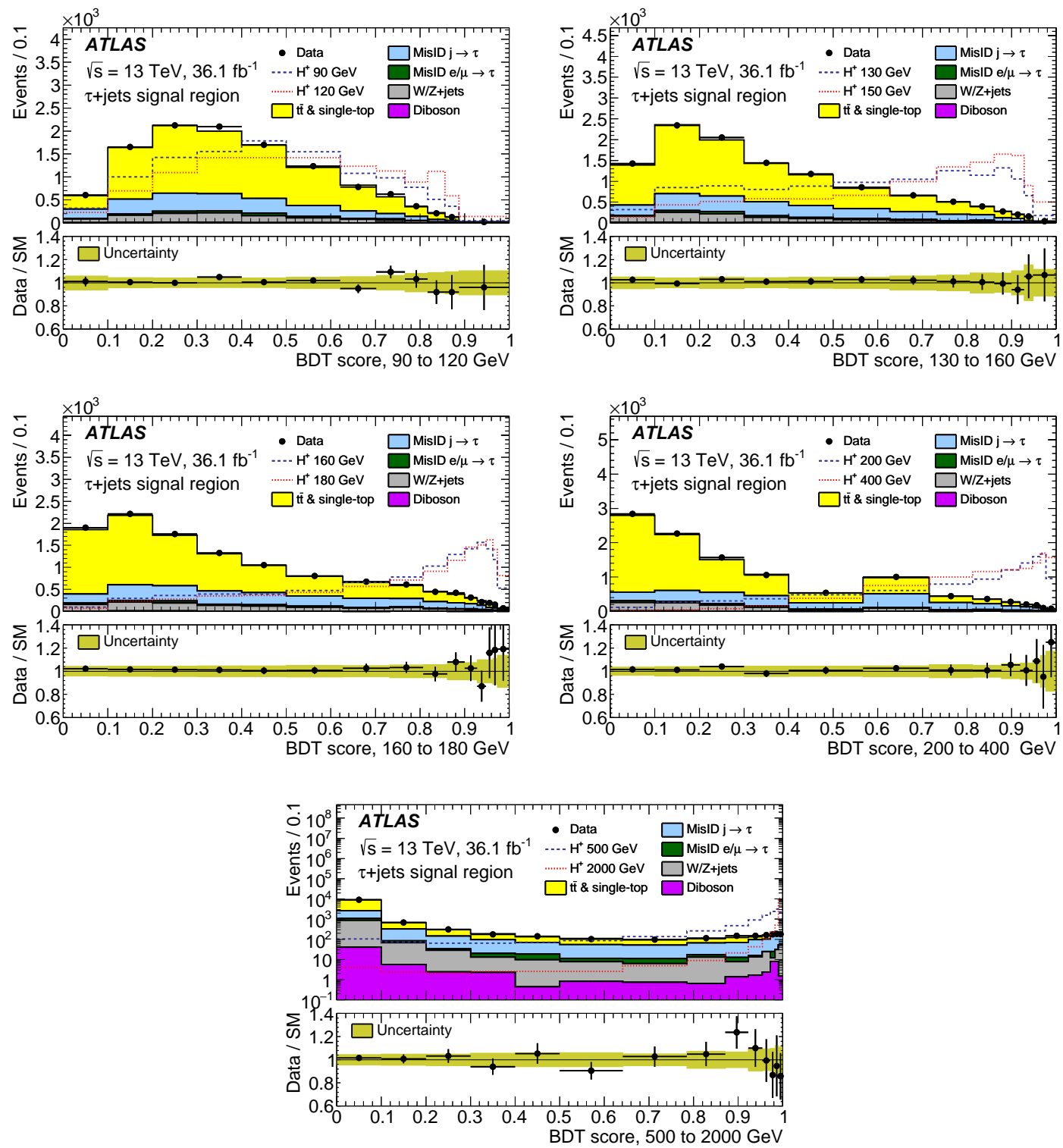

Figure 5: BDTs score distributions in the signal region of the $\tau_{h}+$ jets channel, in the five mass ranges used for the BDTs trainings, after a fit to the data with the background-only hypothesis. The lower panel of each plot shows the ratio of data to the SM background prediction. The uncertainty bands include all statistical and systematic uncertainties. The normalisation of the signal (shown for illustration) corresponds to the integral of the background. 17 

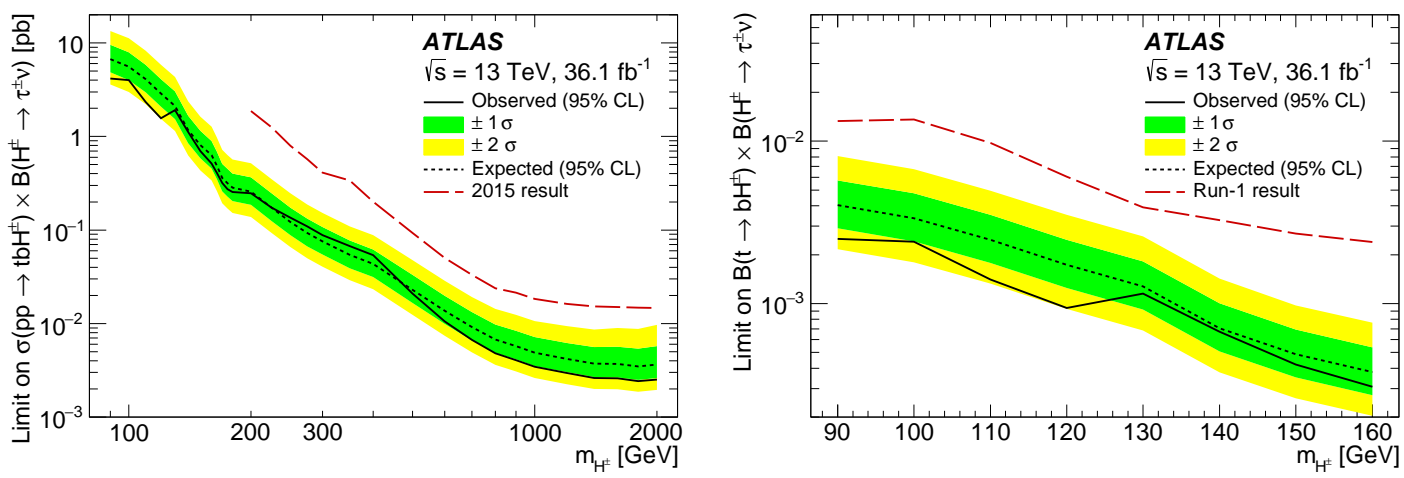

Figure 6: Observed and expected 95\% CL on (left) $\sigma\left(p p \rightarrow t b H^{+}\right) \times B\left(H^{+} \rightarrow \tau \nu\right)$ and (right) $B\left(t \rightarrow b H^{+}\right) \times B\left(H^{+} \rightarrow \tau \nu\right)$ as a function of the charged Higgs boson mass, after combining the $\tau_{h}+$ jets and $\tau_{h}+$ leptons channels. 17

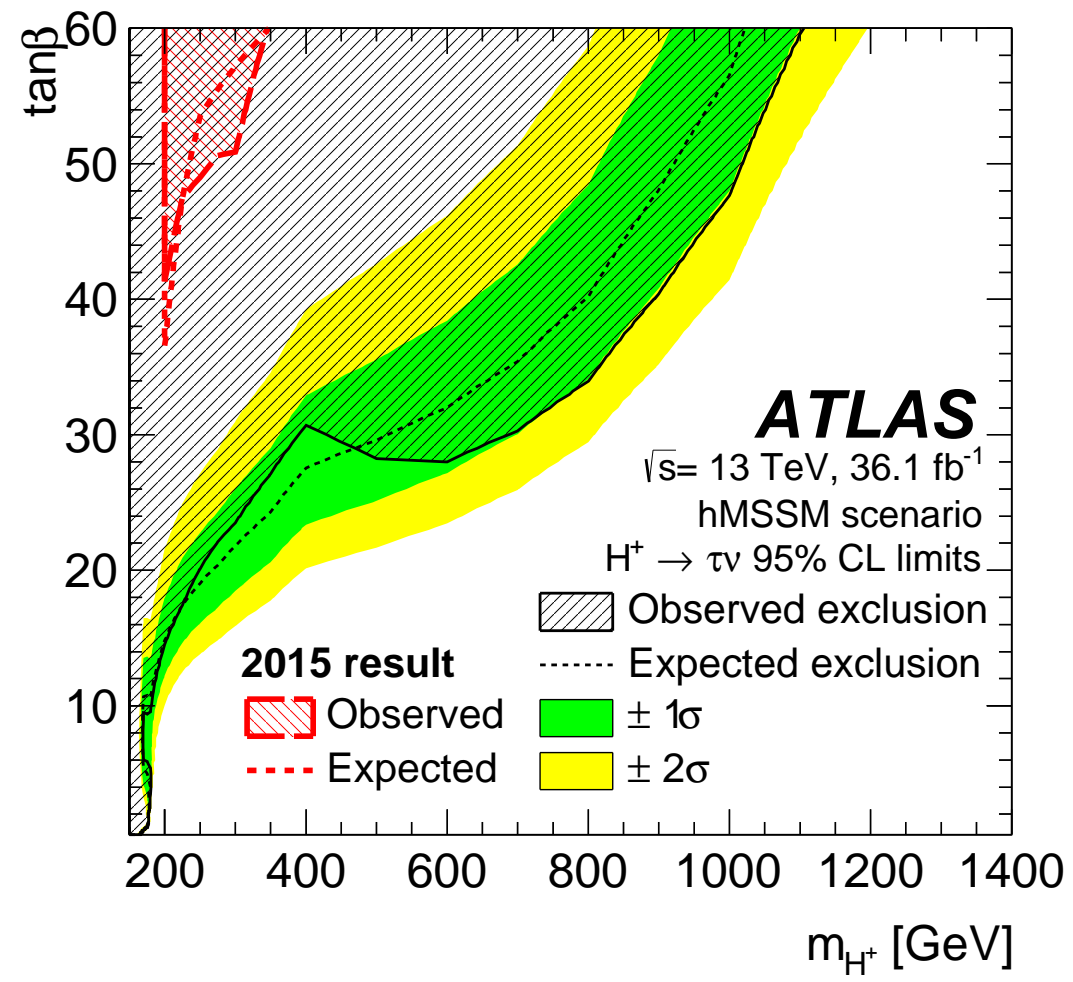

Figure 7: 95\% CL exclusion limits on $\tan \beta$ as a function of the charged Higgs boson mass in the context of the hMSSM scenario, for the regions in which theoretical predictions are available $(0.5 \leq \tan \beta \leq 60)$. 17 


\section{Search for new light bosons in decays of the $h(125)$}

The combination of data collected at center-of-mass energies of 7 and $8 \mathrm{TeV}$ by ATLAS and CMS constrains branching fractions of the Higgs boson to particles beyond the SM to less than $34 \%$ at $95 \%$ CL 19 . Decay chain $h(125) \rightarrow a a$ are allowed in $2 \mathrm{HDM}+\mathrm{S}$ scenarios.

Four different type of $2 \mathrm{HDM}+\mathrm{S}$ forbid flavour-changing neutral current at tree level. In Type-I, all SM particles couple to the first doublet. In Type-II, up-type quarks couple to the first doublet, whereas leptons and down-type quarks couple to the second doublet. NMSSM is a particular case of $2 \mathrm{HDM}+\mathrm{S}$ of Type-II. In Type-III, quarks couple to the first doublet, and leptons to the second one. Finally, in Type-IV, leptons and up-type quarks couple to the first doublet, while down-type quarks couple to the second doublet.

The analysis here presented are based on pp collisions collected in 2016 by the CMS experiment at a center-of-mass energy of $13 \mathrm{TeV}$, corresponding to an integrated luminosity of $35.9 \mathrm{fb}^{-1}$. Decay chains considered are $a a \rightarrow b \bar{b} \tau \tau[20]$ and $a a \rightarrow \mu \mu \tau \tau$ [21]. Masses of the pseudoscalar boson between 15.0 and $62.5 \mathrm{GeV}$ are probed.

\section{$4.1 \quad h \rightarrow a a \rightarrow b \bar{b} \tau \tau$}

Three different $\tau \tau$ final states are considered: $e \mu, e \tau_{h}$, and $\mu \tau_{h}$. They are additionally required to contain at least one b-tagged jet.

To increase the sensitivity of the analysis, events in each final state are separated into four categories with different signal-to-background ratios. The categories are defined on the basis of $m_{\tau \tau b}^{v i s}$, the invariant mass of the visible decay products of the $\tau$ leptons and the b-tagged jet with the highest $p_{T}$. This variable use the difference in the kinematic of the final objects in signal events and background events. Usually, $m_{\tau \tau b}^{v i s}$ has low values for the former and high for latter.

The dominant backgrounds, having these objects in the final state, are $t \bar{t}$ and $Z \rightarrow t t$ production. Another large background consists of events with jets misidentified as $\tau_{h}$, such as $W+$ jets events, the background from SM events composed uniquely of jets produced through the strong interaction, referred to as QCD multijet events, or semileptonic tt events. The misidentified background is estimated through the Fake Rate Method described in 20 .

\section{$4.2 \quad h \rightarrow a a \rightarrow \mu \mu \tau \tau$}

The analysis focus on four different final states that cover the different possible $\tau$ lepton decay modes: $\mu \mu+e \mu, \mu \mu+e \tau_{h}, \mu \mu+\mu \tau_{h}$, and $\mu \mu+\tau_{h} \tau_{h}$. The $\mu \mu+e e$ and $\mu \mu+\mu \mu$ final states are not considered because of their smaller branching fractions and the large background contribution from ZZ production.

The background composed of events where at least one jet is misidentified as one of the final state leptons is estimated from data. Such events include mostly Z+jets and WZ+jets events, but there are also minor contributions from $Z Z \rightarrow 2 l 2 q$ events, $t \bar{t}$ production, or from the background from SM QCD multijet events.

The analysis scans the reconstructed dimuon mass spectrum for a characteristic resonance structure. The event selection and signal extraction used in this analysis are optimized for the $h \rightarrow a a \rightarrow \mu \mu \tau \tau$ decay channel, where h has a mass of $125 \mathrm{GeV}$. Events from the $h \rightarrow a a \rightarrow \tau \tau \tau \tau$ process can also enter the signal region when at least two of the $\tau$ leptons decay leptonically to muons and neutrinos. These events are treated as a part of the signal even if they do not exhibit a narrow dimuon mass peak. 


\subsection{Results}

For the $h \rightarrow a a \rightarrow b b \tau \tau$ decay channel, a global binned maximum-likelihood fit based on the $m_{\tau \tau}^{v i s}$ distributions, in the different channels and categories, is performed for the search for an excess of signal events over the expected background. Unbinned maximumlikelihood fit to the dimuon invariant mass distribution is used in the $h \rightarrow a a \rightarrow \mu \mu \tau \tau$ decay channel.

No significant excess of data is observed above the expected SM background. Upper limits at 95\% CL are set on $\left(\sigma(h) / \sigma_{S M}\right) \times B(h \rightarrow a a \rightarrow \mu \mu \tau \tau)$ and $\left(\sigma(h) / \sigma_{S M}\right) \times B(h \rightarrow$ $a a \rightarrow b b \tau \tau)$ for pseudoscalar masses between 15.0 and $62.5 \mathrm{GeV}$.

Figure 8 shows $95 \%$ CL upper limits obtained from combining the different final state considered in each analysis.
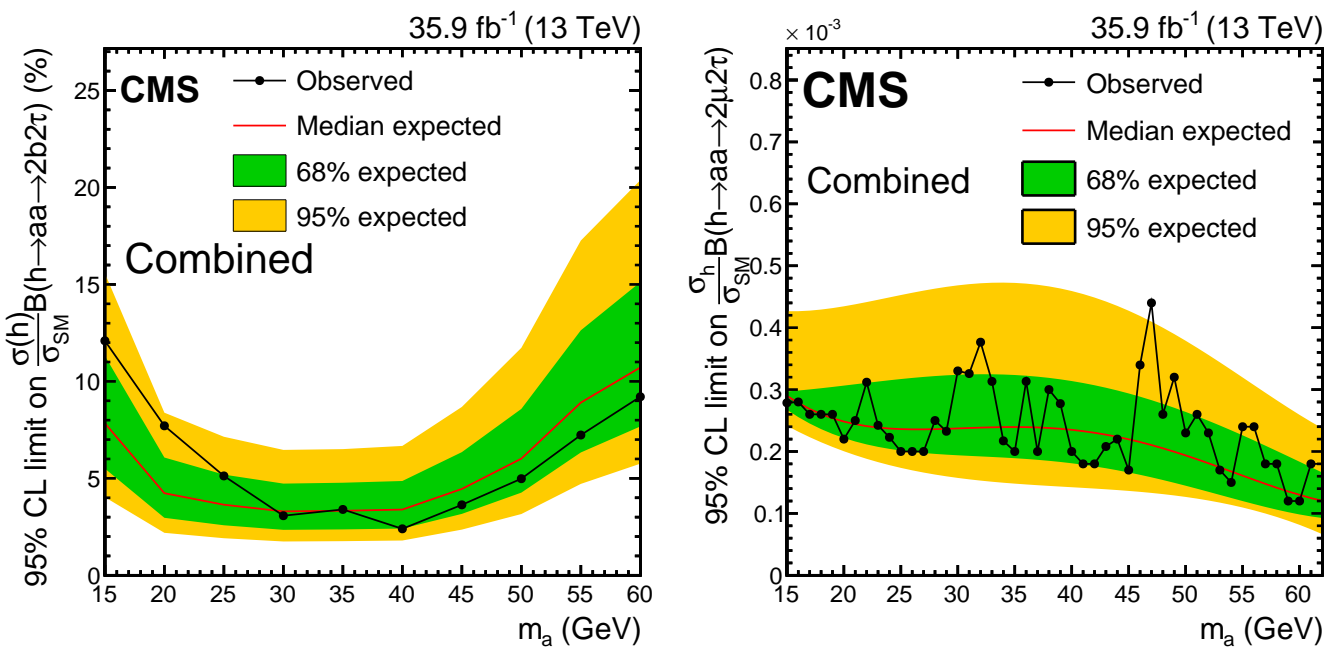

Figure 8: Upper limits at 95\% CL on (left) $\left(\sigma(h) / \sigma_{S M}\right) \times B(h \rightarrow a a \rightarrow b b \tau \tau)$ and on (right) $\left(\sigma(h) / \sigma_{S M}\right) \times B(h \rightarrow a a \rightarrow \mu \mu \tau \tau)$ where the $h \rightarrow a a \rightarrow 4 \tau$ process is considered as a part of the signal, and is scaled with respect to the $h \rightarrow a a \rightarrow \mu \mu \tau \tau$ signal. 20, 21]

This translates to limits on $\left(\sigma(h) / \sigma_{S M}\right) \times B(h \rightarrow a a)$ in the different $2 \mathrm{HDM}+\mathrm{S}$ scenarios. As explained at the beginning of Section 4.2 , the different scenarios are related to how the leptons, up-quark, and down-quark interact with the two doublets introduced. The two analysis have different sensitivity in the $m_{a}-\tan \beta$ plane due to the involvement of down-quarks and leptons in the $b b \tau \tau$ and only leptons in $\mu \mu \tau \tau$. In the Type-I scenario, and Type-II scenario, with $\tan \beta>1$, assuming the SM production cross section and mechanisms for the Higgs boson, limits on $\left(\sigma(h) / \sigma_{S M}\right) \times B(h \rightarrow a a)$ are about down to $20 \%$ for $b b \tau \tau$ and down to $33 \%$ for $\mu \mu \tau \tau$. For Type-III and Type-IV scenarios, the limits are depicted in Figure 9.

\section{Conclusion}

Several searches for BSM Higgs bosons, with tau leptons in the final state, have been carried out in the ATLAS and CMS experiments using 2016 data at $\sqrt{s}=13 \mathrm{TeV}$. No evidence of additional Higgs bosons has been observed. Upper limits are provided on the cross-section times branching fraction for different searches. The results are, furthermore, interpreted in the context of an extended Higgs sector, such as MSSM and NMSSM. The full Run- 2 data, in which the integrated luminosity has reached $\sim 140 \mathrm{fb} b^{1}$ will give an incredible boost to the sensitivity for searches of new physics in the Higgs sector. 


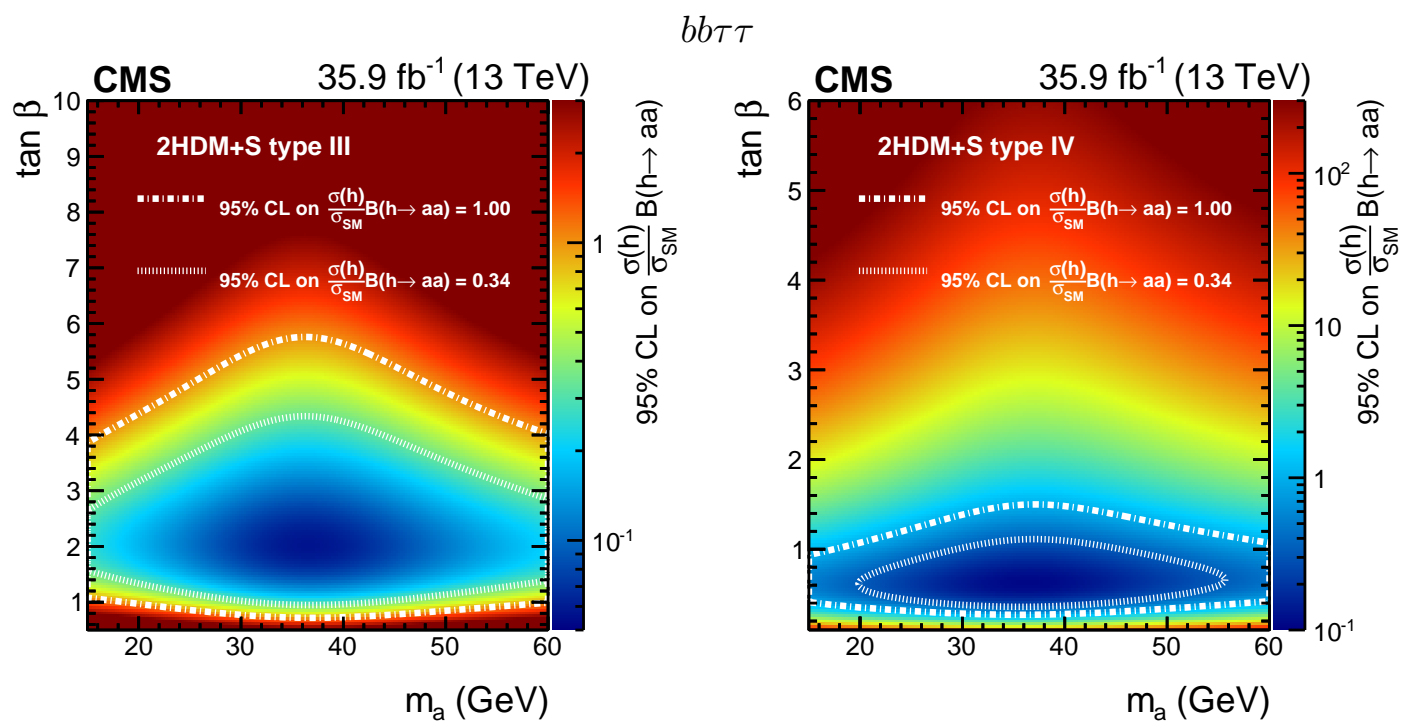

$\mu \mu \tau \tau$
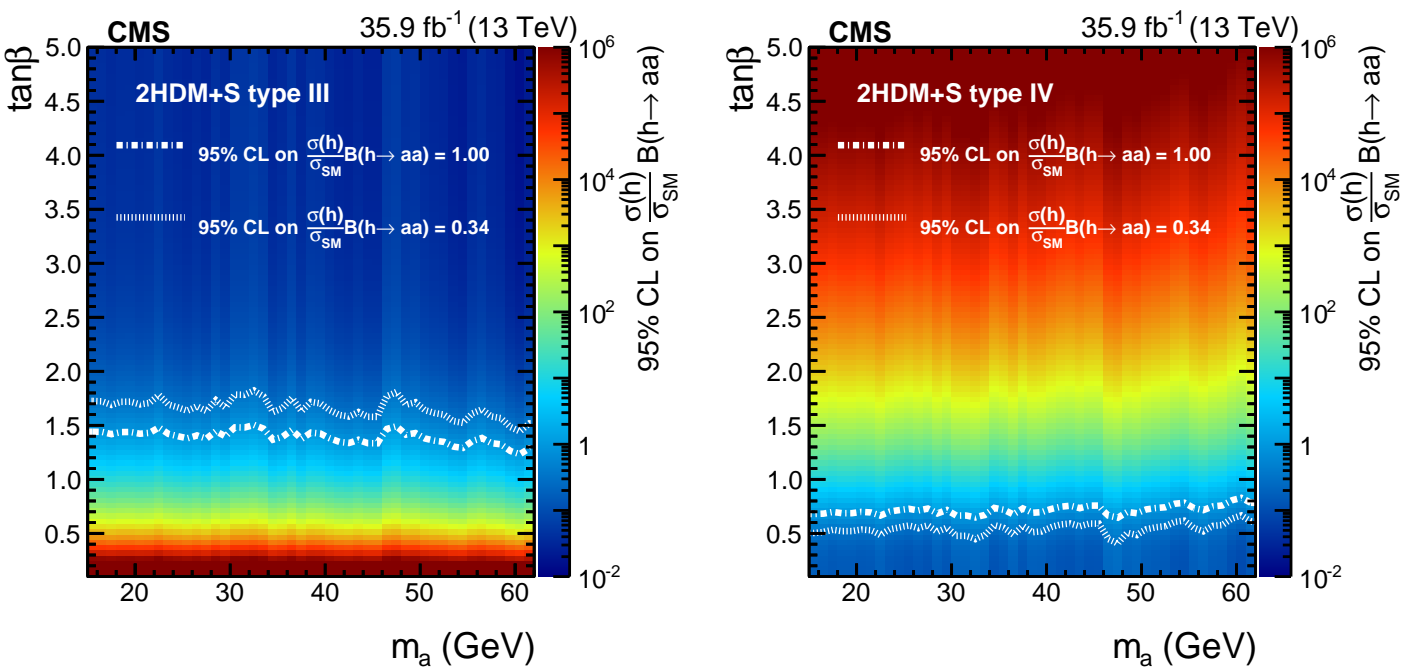

Figure 9: Observed 95\% CL limits on $\left(\sigma(h) / \sigma_{S M}\right) \times B(h \rightarrow a a)$ in $2 \mathrm{HDM}+\mathrm{S}$ of type III (left), and type IV (right). The contours corresponding to a 95\% CL exclusion of $\left(\sigma(h) / \sigma_{S M}\right) \times B(h \rightarrow a a)=1.00$ and 0.34 are drawn with dashed lines. The number $34 \%$ corresponds to the limit on the branching fraction of the Higgs boson to beyond-the-SM particles at the $95 \%$ CL obtained with data collected at center-of-mass energies of 7 and $8 \mathrm{TeV}$ by the ATLAS and CMS experiments [19. 20,21 


\section{References}

[1] G. Aad et al., The ATLAS Experiment at the CERN Large Hadron Collider, JINST 3, S08003 (2008), doi:10.1088/1748-0221/3/08/S08003

[2] S. Chatrchyan et al., The CMS Experiment at the CERN LHC, JINST 3, S08004 (2008), doi:10.1088/1748-0221/3/08/S08004.

[3] G. Aad et al., Observation of a new particle in the search for the Standard Model Higgs boson with the ATLAS detector at the LHC, Phys. Lett. B716, 1 (2012), doi:10.1016/j.physletb.2012.08.020, 1207.7214.

[4] S. Chatrchyan et al., Observation of a new boson at a mass of 125 GeV with the CMS experiment at the LHC, Phys. Lett. B716, 30 (2012), doi:10.1016/j.physletb.2012.08.021, 1207.7235.

[5] S. Chatrchyan et al., Observation of a new boson with mass near $125 \mathrm{GeV}$ in pp collisions at $\sqrt{s}=7$ and 8 TeV, JHEP 06, 081 (2013), doi:10.1007/JHEP06(2013)081, 1303.4571.

[6] Yu. A. Golfand and E. P. Likhtman, Extension of the Algebra of Poincare Group Generators and Violation of p Invariance, JETP Lett. 13, 323 (1971), [Pisma Zh. Eksp. Teor. Fiz.13,452(1971)].

[7] J. Wess and B. Zumino, Supergauge Transformations in Four-Dimensions, Nucl. Phys. B70, 39 (1974), doi:10.1016/0550-3213(74)90355-1, [,24(1974)].

[8] P. Fayet, Supergauge Invariant Extension of the Higgs Mechanism and a Model for the electron and Its Neutrino, Nucl. Phys. B90, 104 (1975), doi:10.1016/05503213(75)90636-7.

[9] P. Fayet, Spontaneously Broken Supersymmetric Theories of Weak, Electromagnetic and Strong Interactions, Phys. Lett. 69B, 489 (1977), doi:10.1016/03702693(77)90852-8.

[10] D. Curtin et al., Exotic decays of the 125 GeV Higgs boson, Phys. Rev. D90(7), 075004 (2014), doi:10.1103/PhysRevD.90.075004, 1312.4992.

[11] M. Aaboud et al., Search for additional heavy neutral Higgs and gauge bosons in the ditau final state produced in $36 \mathrm{fb}^{1}$ of $p p$ collisions at $\sqrt{\mathrm{s}}=13 \mathrm{TeV}$ with the ATLAS detector, JHEP 01, 055 (2018), doi:10.1007/JHEP01(2018)055, 1709.07242.

[12] A. M. Sirunyan et al., Search for additional neutral MSSM Higgs bosons in the $\tau \tau$ final state in proton-proton collisions at $\sqrt{s}=13 \mathrm{TeV}$, JHEP 09, 007 (2018), doi:10.1007/JHEP09(2018)007, 1803.06553.

[13] H. S. S. O. e. a. Carena, M., Mssm higgs boson searches at the lhc: benchmark scenarios after the discovery of a higgs-like particle, Eur. Phys. J. C 73, 2552 (2013), doi:10.1140/epjc/s10052-013-2552-1.

[14] D. de Florian et al., Handbook of LHC Higgs Cross Sections: 4. Deciphering the Nature of the Higgs Sector (2016), doi:10.23731/CYRM-2017-002, 1610.07922.

[15] Summary results of high mass BSM Higgs searches using CMS run-I data, Tech. Rep. CMS-PAS-HIG-16-007, CERN, Geneva (2016). 
[16] C. Degrande, R. Frederix, V. Hirschi, M. Ubiali, M. Wiesemann and M. Zaro, Accurate predictions for charged Higgs production: Closing the $m_{H^{ \pm}} \sim m_{t}$ window, Phys. Lett. B772, 87 (2017), doi:10.1016/j.physletb.2017.06.037, 1607.05291.

[17] M. Aaboud et al., Search for charged Higgs bosons decaying via $H^{ \pm} \rightarrow$ $\tau^{ \pm} \nu_{\tau}$ in the $\tau+$ jets and $\tau+$ lepton final states with $36 \mathrm{fb}^{-1}$ of pp collision data recorded at $\sqrt{s}=13$ TeV with the ATLAS experiment, JHEP 09, 139 (2018), doi:10.1007/JHEP09(2018)139, 1807.07915.

[18] C. Collaboration, Search for charged Higgs bosons with the $H^{ \pm} \rightarrow \tau^{ \pm} \nu_{\tau}$ decay channel in proton-proton collisions at $\sqrt{s}=13 \mathrm{TeV}$ (2018).

[19] G. Aad et al., Measurements of the Higgs boson production and decay rates and constraints on its couplings from a combined ATLAS and CMS analysis of the LHC pp collision data at $\sqrt{s}=7$ and $8 \mathrm{TeV}$, JHEP 08, 045 (2016), doi:10.1007/JHEP08(2016)045, 1606.02266.

[20] A. M. Sirunyan et al., Search for an exotic decay of the Higgs boson to a pair of light pseudoscalars in the final state with two $b$ quarks and two $\tau$ leptons in proton-proton collisions at $\sqrt{s}=13 \mathrm{TeV}$, Phys. Lett. B785, 462 (2018), doi:10.1016/j.physletb.2018.08.057, 1805.10191.

[21] A. M. Sirunyan et al., Search for an exotic decay of the Higgs boson to a pair of light pseudoscalars in the final state of two muons and two $\tau$ leptons in proton-proton collisions at $\sqrt{s}=13 \mathrm{TeV}$ (2018), 1805.04865 . 